ESAIM: PROCEEDINGS AND SURVEYS, September 2014, Vol. 45, p. 400-409

J.-S. Dhersin, Editor

\title{
ABSORBING BOUNDARY CONDITIONS FOR 2D TILTED TRANSVERSE ISOTROPIC ELASTIC MEDIA*
}

\author{
HÉlène BarucQ $^{1}$, Lionel Boillot ${ }^{1}$, Henri Calandra ${ }^{2}$ And Julien Diaz ${ }^{1}$
}

\begin{abstract}
This work deals with the construction of a low-order absorbing boundary condition (ABC) for 2D elliptic TTI media, preserving the system stability. The construction is based on comparing and then connecting the slowness curves for isotropic and elliptic TTI waves. Numerical experiments illustrate the performance of the new $\mathrm{ABC}$. They are performed by integrating the $\mathrm{ABC}$ in a DG formulation of Elastodynamics. When applied in a TTI medium, the new ABC performs well with the same level of accuracy than the standard isotropic ABC set in an isotropic medium. The condition demonstrates also a good robustness when applied for large times of simulation.

Résumé. Ce travail porte sur la construction d'une condition aux limites absorbante (CLA) pour les milieux TTI elliptiques, préservant la stabilité du système. La mise en oeuvre repose sur la comparaison puis la mise en relation des courbes de lenteurs pour les ondes isotropes et TTI elliptiques. Des expériences numériques illustrent la performance de ces nouvelles CLA. Elles sont réalisées en intégrant la CLA dans une formulation de type Galerkin discontinue pour l'Élastodynamique. Pour un milieu TTI, la nouvelle CLA est efficace avec le même niveau de précision que la CLA isotrope standard pour un milieu isotrope. On observe aussi que la condition est robuste en temps long.
\end{abstract}

\section{INTRODUCTION}

The numerical simulation of wave propagation in the Earth is very useful for understanding geophysical phenomena such as earthquakes. It is also a noninvasive and efficient tool to explore limited regions of the subsurface. For instance, numerical waves are used by geophysicists to detect possible stocks of hydrocarbons in regions of the subsurface that are tricky to reach. In this case, it is necessary to couple the wave equations with absorbing boundary conditions (ABC). Another approach is to modify the wave equations within a layer surrounding the computational domain. It is the so-called PML (Perfectly Matched Layer) technique. The use of $\mathrm{ABCs}$ has been suggested long ago $[9,10]$ while the PMLs have emerged later $[5,6]$. In the case of isotropic media, PMLs have clearly demonstrated their supremacy on the ABCs. They are easy to implement and do not generate spurious waves. To achieve the same level of accuracy with ABCs, higher order boundary conditions must be considered and difficulties of construction and implementation occur. Moreover, computational costs are significantly increased compared to those generated by PMLs. However, PMLs suffer from stability problems for different classes of anisotropic media [3], especially in TTI (Titled Transverse Isotropic) environments that are of great interest for oil exploration.

* The authors acknowledge the support by the Inria-Total strategic action DIP (http://dip.inria.fr).

${ }^{1}$ INRIA Bordeaux Sud-Ouest, Equipe projet Magique-3D, LMA - UMR CNRS 5142, Université de Pau et des Pays de l'Adour, Avenue de l'Université, BP 1155, 64013 PAU cedex.

2 TOTAL Exploration \& Production, Depth Imaging and High Performance Computing, Avenue Larribau, 64018 PAU

(C) EDP Sciences, SMAI 2014 
Our work falls into the problem of imaging the subsurface. It is an inverse problem which is difficult to solve because it is nonlinear and ill-posed. Even if recent progresses have been achieved, other approaches are used for industrial purposes where fast and robust algorithms are needed. They do not consist in solving exactly the inverse problem and they generally give approximate solutions which are enough accurate for practitioners. Among them, the Reverse Time Migration (RTM) technique is widely used for seismic imaging. It provides images of the subsurface that are made from time correlations of reflected fields which are generated by artificial acoustic sources propagating inside the region to be explored. From numerical experiments that have been performed by using different numerical methods, it has been observed that this technique is robust to the noise which comes from reflections generated by the boundaries of the computational domain. This is why, in that particular context, it is still interesting to develop $\mathrm{ABCs}$ whose main property is stability. Indeed, if spurious reflections prompted by ABCs can be considered as noise, spurious modes created by PMLs heavily pollute seismograms and the images provided by RTM are unusable.

The purpose of this work is to propose an ABC for TTI media that is easily integrated inside a discontinuous finite element formulation. The Discontinuous Galerkin (DG) method we use has been proposed in [7] for the equations of Elastodynamics formulated as a first order. The construction of the new ABC is mainly based on an algebraic computation which focuses on the slowness curves of the elliptic TTI elastic wave equation. Despite the use of an elliptic framework, the numerical experiments are performed in the general TTI case.

\section{Anisotropic Elastodynamics}

Let $\Omega$ be an open bounded domain of $\mathbb{R}^{2}$. Let $\mathbf{x}=(x, z) \in \Omega$ be the space variable and $t \in[0, T]$ be the time. The elastodynamic system reads as:

$$
\begin{cases}\rho(\mathbf{x}) \partial_{t} v(\mathbf{x}, t) & =\nabla \cdot \underline{\underline{\sigma}}(\mathbf{x}, t) \\ \partial_{t} \underline{\underline{\sigma}}(\mathbf{x}, t) & =\underline{\underline{C}}(\mathbf{x}): \underline{\underline{\epsilon}}(v(\mathbf{x}, t))\end{cases}
$$

where $v:=\left(v_{x}, v_{z}\right)$ denotes the velocity wave field and $\sigma:=\left(\sigma_{x x}, \sigma_{x z}, \sigma_{z z}\right)$ is the stress tensor. The parameter $\rho>0$ stands for the density, $\underline{\underline{C}}$ is the stiffness tensor and $\underline{\underline{\epsilon}}(v)=\frac{1}{2}\left(\vec{\nabla} v+(\vec{\nabla} v)^{T}\right)$ represents the strain tensor. As usual, $\vec{\nabla}$ is the gradient and $\nabla$. denotes the divergence operator.

To produce realistic images of the subsurface from the propagation of waves, it has been demonstrated for instance in $[8,20]$ that anisotropy must be taken into account. In this work, we consider transversely isotropic (TI) media, which can be divided into two groups defined as VTI for vertical TI and TTI for tilted TI. Each medium can be described from the representation of wavefront sets as depicted at Fig. 1.

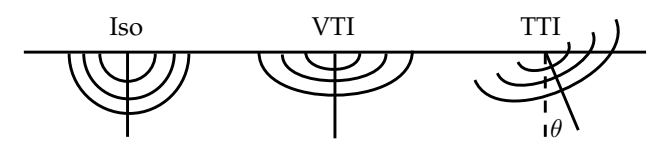

FIGURE 1. Wavefronts for isotropic (left), VTI (center) and TTI (right) media

Obviously, the stiffness tensor $\underline{\underline{C}}$ reads differently in each case:

- Isotropic media are characterized either by the Lamé coefficients $\lambda$ and $\mu$, or by the P-waves and S-waves velocities $V_{p}$ and $V_{s}$ with density $\rho$. Obviously, both characterizations lead to the same entries for $\underline{\underline{C}}$.

- VTI media are defined likewise by $V_{p}, V_{s}$ and $\rho$, and in addition their characterization involves Thomsen [18] VTI parameters $\varepsilon$ and $\delta$. The stencil of $\underline{\underline{C}}$ is then the same than in the isotropic case

- TTI media are described from VTI ones by applying a rotation of angle $\theta$. It is worth noting that $\underline{\underline{C}}$ is dense while it admits null entries in the two previous cases. 


\section{TI FORMULATION}

The general stiffness tensor containing the elasticity is fourth-rank. It can be rewritten in a matricial formulation with the Voigt notation. In $2 \mathrm{D}$, matrix indices $\{1,3,5\}$ refer respectively to tensor indices $\{x x, z z, x z\}$ and the matrix of the stiffness tensor reads as:

$$
C=\left[\begin{array}{lll}
C_{11} & C_{13} & C_{15} \\
C_{13} & C_{33} & C_{35} \\
C_{15} & C_{35} & C_{55}
\end{array}\right]
$$

In the isotropic and the VTI cases, this tensor becomes sparse with $C_{15}=C_{35}=0$, otherwise it is dense.

In the isotropic case, the entries can be defined similarly from the Lamé coefficients $\lambda$ and $\mu$ as follows:

$$
C_{11}=\lambda+2 \mu, C_{33}=\lambda+2 \mu, C_{55}=\mu, C_{13}=\lambda
$$

or from the velocities $V_{p}$ and $V_{s}$, and the density $\rho$

$$
C_{11}=\rho V_{p}^{2}, C_{33}=\rho V_{p}^{2}, C_{55}=\rho V_{s}^{2}, C_{13}=\rho\left(V_{p}^{2}-2 V_{s}^{2}\right)
$$

In the VTI case, the coefficients involve now Thomsen's coefficients [18] $\varepsilon$ and $\delta$ :

$$
\begin{gathered}
C_{11}=\rho V_{p}^{2}(1+2 \varepsilon), C_{33}=\rho V_{p}^{2}, C_{55}=\rho V_{s}^{2}, \\
C_{13}=\rho\left(\sqrt{\left(V_{p}^{2}-V_{s}^{2}\right)^{2}+2 \delta V_{p}^{2}\left(V_{p}^{2}-V_{s}^{2}\right)}-V_{s}^{2}\right)
\end{gathered}
$$

The TTI formulation is more tricky to introduce. The tensor $\underline{\underline{C}}$ is dense (but still symmetrical). It results from the $\theta$-rotation of the VTI formulation, that is:

$$
\underline{\underline{C}}_{i j k l}^{T T I}=\sum_{p} \sum_{q} \sum_{r} \sum_{s} R_{p i} R_{q j} R_{r k} R_{s l} \underline{\underline{C}}_{p q r s}^{V T I}, \quad \text { with } R=\left(\begin{array}{cc}
\cos \theta & -\sin \theta \\
\sin \theta & \cos \theta
\end{array}\right)
$$

Elastodynamics is then governed by wave equations which can be rewritten as the following first order system:

$$
\left\{\begin{array}{l}
\rho \partial_{t} v_{x}=\partial_{x} \sigma_{x x}+\partial_{z} \sigma_{x z} \\
\rho \partial_{t} v_{z}=\partial_{x} \sigma_{x z}+\partial_{z} \sigma_{z z} \\
\partial_{t} \sigma_{x x}=C_{11} \partial_{x} v_{x}+C_{13} \partial_{z} v_{z}+C_{15} \partial_{x} v_{z}+C_{15} \partial_{z} v_{x} \\
\partial_{t} \sigma_{z z}=C_{13} \partial_{x} v_{x}+C_{33} \partial_{z} v_{z}+C_{35} \partial_{x} v_{z}+C_{35} \partial_{z} v_{x} \\
\partial_{t} \sigma_{x z}=C_{15} \partial_{x} v_{x}+C_{35} \partial_{z} v_{z}+C_{55} \partial_{x} v_{z}+C_{55} \partial_{z} v_{x}
\end{array}\right.
$$

\section{Absorbing Boundary Condition (ABC)}

A rigorous methodology for the construction of ABCs is based on the diagonalization of the system (1). This approach has been proposed by Enquist and Majda [9] for strongly hyperbolic systems. It provides a very elegant process for the derivation of ABCs on arbitrarily shaped boundaries [1]. Nevertheless, in practice, it can quickly become uneasy to use because of coupling terms that are difficult to handle in the first stage of the diagonalization. The coupling indeed results in eigenvalues which are difficult to exploit for the construction of efficient ABCs. For instance, the VTI eigenvalues have the general form [12]:

$$
\lambda_{P / S}=\sqrt{\alpha k^{2}-\beta \rho w^{2} \pm \sqrt{\gamma k^{4}-\eta \rho k^{2} w^{2}+\xi \rho^{2} w^{4}}}
$$


where $k$ denotes the frequency related to the time variable by a Fourier transform, and $\alpha, \beta, \gamma, \eta, \xi$ are parameters depending on the tensor coefficients.

From a practical point of view, it is obvious that the numerical handling of $\lambda$ is not feasible because of the composition of two square roots and are uneasy to localize. However, it remains possible to construct a low-order VTI ABC by using a Taylor expansion of order one. Then, focusing on normal incident waves allows the elimination of the derivative terms in the other direction, as described in $[11,13]$ for the isotropic case. This leads, for a boundary with an outward normal $e_{x}$, to the low-order VTI ABC:

$$
\left\{\begin{array}{l}
\sigma_{x x}=\rho V_{p} \sqrt{1+2 \varepsilon} v_{x} \\
\sigma_{x z}=\rho V_{s} v_{z}
\end{array}\right.
$$

which is identical to the first-order ABC obtained by a different way in [2].

Setting $\varepsilon$ to zero in the VTI ABC (4) is identical to the low-order isotropic ABC (e.g. in [19]):

$$
\left\{\begin{array}{l}
\sigma_{x x}=\rho V_{p} v_{x} \\
\sigma_{x z}=\rho V_{s} v_{z}
\end{array}\right.
$$

These simple ABCs can also be obtained by uncoupling the P-waves and the S-waves in the elastic system (1), which means fixing $V_{s}$ or $V_{p}$ to zero. Thus, the Engquist-Majda methodology can be applied to the resulting pseudo-acoustic systems, leading to the first equation of (5) for the P-waves while the second relation in (5) is for the S-waves.

Now, when addressing the case of a TTI medium, the stiffness tensor is dense and Engquist-Majda methodology becomes very tricky to apply. It is even not obvious that the corresponding eigenvalues can be computed explicitly, even for particular TTI media or for uncoupled waves. We have tried to approximate them with the help of Maple ${ }^{(1}$ software. We got results that are actually unusable. This is why we have decided to adopt another approach which is described in the next section.

\section{Elliptic TTI LOW-ORDER ABC}

\subsection{Slowness curves}

Our approach is based on the slowness curves for TTI media. This idea has been already suggested by Savadatti and Guddati who published four interesting articles [14-17] about revisited ABC involving arguments that are usually claimed for PMLs analysis. In particular, they provide a characterization of different types of anisotropy by involving slowness curves. In the same time, slowness curves have been introduced in [4] to construct $\mathrm{ABCs}$ for anisotropic acoustic equations.

In this work, we propose to follow the same ideas than in [4] to construct a low-order ABC for waves propagation in TTI media. We restrict our study to the case where $\varepsilon=\delta$ which corresponds to the so-called elliptic TI case. Let us remark that low-order VTI ABCs do not depend on the TI- $\delta$ parameter, it might be possible that the simplest TTI ABCs do not involve $\delta$ either. Hence, the elliptic case could give rise to an efficient low-order $\mathrm{ABC}$ for more general cases.

For the construction of the ABC, we thus begin by addressing the form of the slowness curves. Fig. 2 depicts these profiles for an isotropic and an elliptic TTI elastic system. It clearly appears that the S-waves slowness curves are identical in both cases, forming a circle, whereas the P-waves slowness curve is circular in the isotropic case and elliptical in the elliptic TTI case.

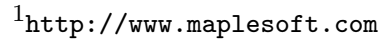



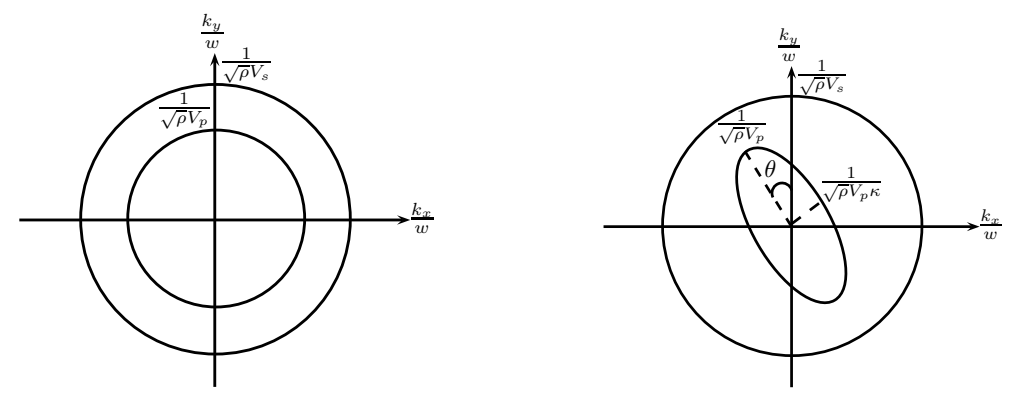

FIGURE 2. Slowness curves of isotropic (left) and elliptic TTI (right) elasticity

Let us tackle the construction of the $\mathrm{ABC}$ by choosing the isotropic case as a reference. That makes sense since isotropic $\mathrm{ABCs}$ are stable and we are mainly interested in stable boundary conditions. We then propose to find auxiliary fields $\left(v^{*}, \sigma^{*}\right)$ which are solutions to an isotropic wave equation and to establish a link with $(v, \sigma)$. By this way, we will be able to deduce the boundary condition that is satisfied by $(v, \sigma)$ when $\left(v^{*}, \sigma^{*}\right)$ satisfies the simplest $\mathrm{ABC}$ for isotropic elastic waves.

In, Fig. 2, frequencies $k_{x}^{*}$ and $k_{z}^{*}$ are the symbols of the derivatives $\partial_{x}^{*}$ and $\partial_{z}^{*}$ of an isotropic elastic system. In the same way, the partial derivatives $\partial_{x}$ and $\partial_{z}$ of an elliptic TTI elastic system are related to $k_{x}$ and $k_{z}$. So, we can join $\partial_{x}^{*}$ and $\partial_{z}^{*}$ with $\partial_{x}$ and $\partial_{z}$ by constructing a map which transforms the slowness curve forms.

\subsection{An elliptic TTI ABC}

Let us focus on P-waves only, i.e. setting $V_{s}$ to zero in (1). The construction of the elliptic TTI P-waves $\mathrm{ABC}$ follows a multi-step procedure.

Step 1 consists in elaborating a change of variables which modifies a rotated ellipse into a circle. According to Fig. 2 , we denote by $\left(k_{x}, k_{z}\right)$ the slowness variables for the TTI case and $\left(k_{x}^{\star}, k_{z}^{\star}\right)$ corresponds to the isotropic case. We then seek coefficients $\left\{\mu_{j}\right\}, 1 \leqslant j \leqslant 4$, such that:

$$
k_{x}=\mu_{1} k_{x}^{\star}+\mu_{2} k_{z}^{\star} \quad \text { and } \quad k_{z}=\mu_{3} k_{x}^{\star}+\mu_{4} k_{z}^{\star}
$$

Let us consider the case where the $\mathrm{ABC}$ acts on a boundary with an outward normal $e_{x}$. Then, the change of coordinates should not impact on the $z$-derivative and $\mu_{3}=0$. In the same way, we could have considered the case where the $\mathrm{ABC}$ is set upon a boundary with an outward normal $e_{z}$. Then, we would have fixed $\mu_{3}=0$.

First, $\left(k_{x}^{\star}, k_{z}^{\star}\right)$ satisfies the quadratic equation

$$
\rho V_{p}^{2} k_{x}^{\star 2}+\rho V_{p}^{2} k_{z}^{\star 2}=1
$$

Second, $\left(k_{x}, k_{z}\right)$ satisfies the generic relation

$$
\xi_{1} k_{x}^{2}+\xi_{2} k_{x} k_{z}+\xi_{3} k_{z}^{2}=1
$$

with $\kappa=1+2 \varepsilon$, and

$$
\left\{\begin{array}{l}
\xi_{1}=\rho V_{p}^{2}\left(\kappa^{2} \cos ^{2} \theta+\sin ^{2} \theta\right) \\
\xi_{2}=2 \cos \theta \sin \theta \rho V_{p}^{2}\left(\kappa^{2}-1\right) \\
\xi_{3}=\rho V_{p}^{2}\left(\kappa^{2} \sin ^{2} \theta+\cos ^{2} \theta\right)
\end{array}\right.
$$


By plugging (6) into (8), we then get:

$$
\xi_{1} \mu_{1}^{2} k_{x}^{\star 2}+\mu_{1}\left(2 \xi_{1} \mu_{2}+\xi_{2} \mu_{4}\right) k_{x}^{\star} k_{z}^{\star}+\left(\xi_{1} \mu_{2}^{2}+\xi_{2} \mu_{2} \mu_{4}+\xi_{3} \mu_{4}^{2}\right) k_{z}^{\star 2}=1
$$

Then, by identifying the coefficients of the above equation with the coefficients of (7), we obtain:

$$
\left\{\begin{array}{l}
\mu_{1}=\sqrt{1 /\left(\kappa^{2} \cos ^{2} \theta+\sin ^{2} \theta\right)} \\
\mu_{2}=-\left(\kappa^{2}-1\right) \cos \theta \sin \theta / \kappa \sqrt{\kappa^{2} \cos ^{2} \theta+\sin ^{2} \theta} \\
\mu_{4}=\frac{1}{\kappa} \sqrt{\kappa^{2} \cos ^{2} \theta+\sin ^{2} \theta}
\end{array}\right.
$$

Now, we use (7) to deduce: :

$$
\partial_{x}=\mu_{1} \partial_{x}^{\star}+\mu_{2} \partial_{z}^{\star} \quad \text { and } \quad \partial_{z}=\mu_{4} \partial_{z}^{\star}
$$

Step 2 addresses the corresponding change for the velocity field $v$. Following step 1 , we define $\left(v_{x}^{\star}, v_{z}^{\star}\right)$ as the coordinates of $v=\left(v_{x}, v_{z}\right)$ in the set of isotropic variables. We seek them as follows:

$$
\left\{\begin{array}{l}
v_{x}=\alpha_{1} v_{x}^{\star}+\alpha_{2} v_{z}^{\star} \\
v_{z}=\alpha_{3} v_{x}^{\star}+\alpha_{4} v_{z}^{\star}
\end{array}\right.
$$

Then we have to find coefficients $\left\{\alpha_{j}\right\}_{1 \leqslant j \leqslant 4}$ in such a way that $\left(v_{x}^{\star}, v_{z}^{\star}\right)$ is solution of the isotropic elastic wave system:

$$
\left\{\begin{array}{l}
\frac{1}{\rho V_{p}^{2}} \rho \partial_{t}^{2} v_{x}^{\star}=\partial_{x}^{\star 2} v_{x}^{\star}+\partial_{x}^{\star} \partial_{z}^{\star} v_{z}^{\star} \\
\frac{1}{\rho V_{p}^{2}} \rho \partial_{t}^{2} v_{z}^{\star}=\partial_{z}^{\star} \partial_{x}^{\star} v_{x}^{\star}+\partial_{z}^{\star 2} v_{z}^{\star}
\end{array}\right.
$$

To simplify the computations, it is easier to uncouple $v$ from $\sigma$ by considering the second-order formulation of Elastodynamics:

$$
\left\{\begin{aligned}
\rho \partial_{t}^{2} v_{x} & =C_{11} \partial_{x}^{2} v_{x}+C_{12} \partial_{x} \partial_{z} v_{z}+C_{13}\left(\partial_{x}^{2} v_{z}+\partial_{z} \partial_{x} v_{x}\right) \\
& +C_{13} \partial_{x} \partial_{z} v_{x}+C_{23} \partial_{z}^{2} v_{z}+C_{33}\left(\partial_{x} \partial_{z} v_{z}+\partial_{z}^{2} v_{x}\right) \\
\rho \partial_{t}^{2} v_{z} & =C_{13} \partial_{x}^{2} v_{x}+C_{23} \partial_{x} \partial_{z} v_{z}+C_{33}\left(\partial_{x}^{2} v_{z}+\partial_{z} \partial_{x} v_{x}\right) \\
& +C_{12} \partial_{x} \partial_{z} v_{x}+C_{22} \partial_{z}^{2} v_{z}+C_{23}\left(\partial_{x} \partial_{z} v_{z}+\partial_{z}^{2} v_{x}\right)
\end{aligned}\right.
$$

We can then plug (12) and (13) into (15) and identify the coefficients of the resulting system with the ones of (14). In this elliptic TTI case, with $V_{s}=0$, the $\underline{\underline{C}}$ tensor coefficients are easily computable.

After a series of algebraic handlings, we obtain three equations by identifying respectively the operators $\partial_{x}^{\star 2}$, $\partial_{z}^{\star 2}$ and $\partial_{x}^{\star} \partial_{z}^{\star}$. They read as:

$$
\begin{gathered}
\frac{1}{\alpha_{1} \alpha_{4}-\alpha_{2} \alpha_{3}}\left(\begin{array}{cc}
\alpha_{4} & -\alpha_{2} \\
-\alpha_{3} & \alpha_{1}
\end{array}\right) \mu_{1}^{2}\left(\begin{array}{cc}
a^{2} & a b \\
a b & b^{2}
\end{array}\right)\left(\begin{array}{cc}
\alpha_{1} & \alpha_{2} \\
\alpha_{3} & \alpha_{4}
\end{array}\right)=\left(\begin{array}{cc}
\rho V_{p}^{2} & 0 \\
0 & 0
\end{array}\right) \\
\frac{1}{\alpha_{1} \alpha_{4}-\alpha_{2} \alpha_{3}}\left(\begin{array}{cc}
\alpha_{4} & -\alpha_{2} \\
-\alpha_{3} & \alpha_{1}
\end{array}\right) \mu_{1}^{2}\left(\begin{array}{cc}
b^{2} & -a b \\
-a b & a^{2}
\end{array}\right)\left(\begin{array}{ll}
\alpha_{1} & \alpha_{2} \\
\alpha_{3} & \alpha_{4}
\end{array}\right)=\left(\begin{array}{cc}
0 & 0 \\
0 & \rho V_{p}^{2}
\end{array}\right) \\
\frac{1}{\alpha_{1} \alpha_{4}-\alpha_{2} \alpha_{3}}\left(\begin{array}{cc}
\alpha_{4} & -\alpha_{2} \\
-\alpha_{3} & \alpha_{1}
\end{array}\right)\left(\begin{array}{cc}
c & d \\
d & -c
\end{array}\right)\left(\begin{array}{ll}
\alpha_{1} & \alpha_{2} \\
\alpha_{3} & \alpha_{4}
\end{array}\right)=\left(\begin{array}{cc}
0 & \rho V_{p}^{2} \\
\rho V_{p}^{2} & 0
\end{array}\right)
\end{gathered}
$$


with:

$$
\left\{\begin{array}{l}
a=\rho V_{p}\left(\kappa \cos ^{2} \theta+\sin ^{2} \theta\right) / \sqrt{\kappa^{2} \cos ^{2} \theta+\sin ^{2} \theta} \\
b=\rho V_{p}(\kappa-1) \cos \theta \sin \theta / \sqrt{\kappa^{2} \cos ^{2} \theta+\sin ^{2} \theta} \\
c=2 a^{2} \cdot \mu_{2} / \mu_{1}+2 a b \cdot \mu_{4} / \mu_{1} \\
d=b^{2} \cdot \mu_{4} / \mu_{1}+2 a b \cdot \mu_{2} / \mu_{1}+C_{12} \cdot \mu_{1} \mu_{4}
\end{array}\right.
$$

which simplifies to:

$$
\left\{\begin{array}{l}
\alpha_{1}=\frac{a}{b} \alpha_{3} \\
\alpha_{4}=\alpha_{1} \\
\alpha_{2}=-\alpha_{3}
\end{array}\right.
$$

Choosing $\alpha_{1}=1$, we finally get:

$$
v_{x}=v_{x}^{\star}-\frac{b}{a} v_{z}^{\star} \quad \text { and } \quad v_{z}=\frac{b}{a} v_{x}^{\star}+v_{z}^{\star}
$$

Step 3 deals with $\sigma$ which has been avoided at step 2 by working directly on $v$. Following ideas of step 2, we introduce $\sigma^{\star}$ corresponding to $v^{\star}$ and we seek $\left\{\beta_{j}\right\}_{1 \leqslant j \leqslant 9}$ such that

$$
\left\{\begin{array}{l}
\sigma_{x x}=\beta_{1} \sigma_{x x}^{\star}+\beta_{2} \sigma_{z z}^{\star}+\beta_{3} \sigma_{x z}^{\star} \\
\sigma_{z z}=\beta_{4} \sigma_{x x}^{\star}+\beta_{5} \sigma_{z z}^{\star}+\beta_{6} \sigma_{x z}^{\star} \\
\sigma_{x z}=\beta_{7} \sigma_{x x}^{\star}+\beta_{8} \sigma_{z z}^{\star}+\beta_{9} \sigma_{x z}^{\star}
\end{array}\right.
$$

According to the isotropic case, we know that $\sigma_{x x}^{\star}=\sigma_{z z}^{\star}=p^{\star}$ and $\sigma_{x z}^{\star}=0$. We thus have

$$
\left\{\begin{array}{l}
\sigma_{x x}=\left(\beta_{1}+\beta_{2}\right) p^{\star} \\
\sigma_{z z}=\left(\beta_{4}+\beta_{5}\right) p^{\star} \\
\sigma_{x z}=\left(\beta_{7}+\beta_{8}\right) p^{\star}
\end{array}\right.
$$

Thus the problem reduces to find three coefficients denoted by $\left\{\widetilde{\beta}_{j}\right\}_{1 \leqslant j \leqslant 3}$ such that

$$
\sigma_{x x}=\widetilde{\beta_{1}} p^{\star}, \sigma_{z z}=\widetilde{\beta_{2}} p^{\star}, \sigma_{x z}=\widetilde{\beta_{3}} p^{\star}
$$

Next we proceed as in step 2 by identifying the coefficients of the system satisfied by $\sigma^{\star}$. We then get:

$$
\left\{\begin{array}{l}
\widetilde{\beta_{1}}=\sqrt{\kappa^{2} \cos ^{2} \theta+\sin ^{2} \theta} \\
\widetilde{\beta_{2}}=\left(\cos ^{2} \theta+\kappa \sin ^{2} \theta\right) \sqrt{\kappa^{2} \cos ^{2} \theta+\sin ^{2} \theta} /\left(\kappa \cos ^{2} \theta+\sin ^{2} \theta\right) \\
\widetilde{\beta_{3}}=(\kappa-1) \cos \theta \sin \theta \sqrt{\kappa^{2} \cos ^{2} \theta+\sin ^{2} \theta} /\left(\kappa \cos ^{2} \theta+\sin ^{2} \theta\right)
\end{array}\right.
$$

Step 4, which ends the multi-step procedure of construction, consists in using the isotropic ABC in (5) and in applying the change of coordinates described in the relations (21) and (24). For a boundary with an outward normal $e_{x}$, the $\mathrm{P}$-waves contribution of the isotropic $\mathrm{ABC}$ is $p^{\star}=-\rho V_{p} v_{x}^{\star}$. In this case, $\sigma_{x x}^{\star}=p^{\star}$. And so the elliptic TTI P-waves ABC is obtained with the coefficients in (19) and (25):

$$
\left\{\begin{array}{l}
\sigma_{x x}=-\rho V_{p} \frac{\kappa \cos ^{2} \theta+\sin ^{2} \theta}{\sqrt{\kappa^{2} \cos ^{2} \theta+\sin ^{2} \theta}}\left[\left(\kappa \cos ^{2} \theta+\sin ^{2} \theta\right) v_{x}+(\kappa-1) \cos \theta \sin \theta v_{z}\right] \\
\sigma_{z x}=-\rho V_{p} \frac{(\kappa-1) \cos \theta \sin \theta}{\sqrt{\kappa^{2} \cos ^{2} \theta+\sin ^{2} \theta}}\left[\left(\kappa \cos ^{2} \theta+\sin ^{2} \theta\right) v_{x}+(\kappa-1) \cos \theta \sin \theta v_{z}\right]
\end{array}\right.
$$


We have focused on P-waves and constructed a low-order ABC. We did not address the case of S-waves since their slowness curve is identical in the elliptic TTI case as in the isotropic case. Hence, our approach does not impact on the S-waves. Moreover, as previously noticed, the low-order ABC can be viewed as the addition of $\mathrm{P}$-waves and S-waves contributions. Therefore, for the S-waves, we decided to apply the low-order ABC in (5), obtained in an isotropic media. Finally, for a boundary with an outward normal $e_{x}$, the complete low-order elliptic TTI ABC reads as:

$$
\left\{\begin{array}{l}
\sigma_{x x}=-\rho V_{p} \frac{\kappa \cos ^{2} \theta+\sin ^{2} \theta}{\sqrt{\kappa^{2} \cos ^{2} \theta+\sin ^{2} \theta}}\left[\left(\kappa \cos ^{2} \theta+\sin ^{2} \theta\right) v_{x}+(\kappa-1) \cos \theta \sin \theta v_{z}\right] \\
\sigma_{z x}=-\rho V_{p} \frac{(\kappa-1) \cos \theta \sin \theta}{\sqrt{\kappa^{2} \cos ^{2} \theta+\sin ^{2} \theta}}\left[\left(\kappa \cos ^{2} \theta+\sin ^{2} \theta\right) v_{x}+(\kappa-1) \cos \theta \sin \theta v_{z}\right]-\rho V_{s} v_{z}
\end{array}\right.
$$

\section{Stability of the ABC}

We know that $\rho>0$ and $\underline{\underline{C}}$ is symmetrical and positive, i.e. $(\underline{\underline{C}}: \underline{\underline{\xi}}) \cdot \underline{\underline{\xi}}>0 \forall \underline{\underline{\xi}}$ symmetrical. $\underline{\underline{C}}$ is thus invertible and let us denote by $\underline{\underline{C}}^{-1}$ its inverse, which is also positive.

Let us consider a domain $\left.\Omega_{x}=\right]-\infty, 0[\times]-\infty,+\infty\left[\right.$ and $\Gamma_{x}$ denotes its external boundary. Let us introduce the functional $\mathbb{E}(t)=\frac{1}{2}\left(\|v\|_{\Omega_{x}, \rho}^{2}+\|\underline{\underline{\sigma}}\|_{\Omega_{x}, \underline{\underline{C}}^{-1}}^{2}\right)$. Then, formally, we have:

$$
\frac{d}{d t} \mathbb{E}(t)=\int_{\Omega_{x}} \rho \partial_{t} v v d \mathbf{x}+\int_{\Omega_{x}} \partial_{t} \underline{\underline{\sigma}}:\left(\underline{\underline{C}}^{-1}: \underline{\underline{\sigma}}\right) d \mathbf{x}=\int_{\Gamma_{x}} \sigma_{x x} v_{x}+\sigma_{x z} v_{z} d \mathbf{x}
$$

Now, we can replace the first term of (28) by using the first equation of the elastodynamics system (1) and a generalized Green formula:

$$
\int_{\Omega_{x}} \rho \partial_{t} v v d \mathbf{x}=\int_{\Omega_{x}} \nabla \cdot \underline{\underline{\sigma}} v d \mathbf{x}=-\int_{\Omega_{x}} \underline{\underline{\sigma}}: \vec{\nabla} v d \mathbf{x}+\int_{\Gamma_{x}}(\underline{\underline{\sigma} \mathbf{n}}) v d \mathbf{x}
$$

We can also replace the second term of (28) by using the second equation of the elastodynamics system (1):

$$
\int_{\Omega_{x}} \partial_{t} \underline{\underline{\sigma}}:\left(\underline{\underline{C}}^{-1}: \underline{\underline{\sigma}}\right) d \mathbf{x}=\int_{\Omega_{x}}[\underline{\underline{C}}: \underline{\underline{\epsilon}}(v)]:\left(\underline{\underline{C}}^{-1}: \underline{\underline{\sigma}}\right) d \mathbf{x}=\int_{\Omega_{x}} \underline{\underline{\sigma}}: \vec{\nabla} v d \mathbf{x}
$$

Finally, using that $\mathbf{n}$ is the outward normal to $\Gamma_{x}$, i.e. $\left(n_{x}=1 ; n_{z}=0\right), \underline{\underline{\sigma}}=\left(\sigma_{x x}, \sigma_{x z}\right)$, and replacing by the $\mathrm{ABC}(27)$, equation (28) reduces to:

$$
\frac{d}{d t} \mathbb{E}(t)=\int_{\Gamma_{x}} \underline{\underline{\sigma}} \mathbf{n} d \mathbf{x}=\int_{\Gamma_{x}}-\frac{\rho V_{p}}{\sqrt{\kappa^{2} \cos ^{2} \theta+\sin ^{2} \theta}}\left[\left(\kappa \cos ^{2} \theta+\sin ^{2} \theta\right) v_{x}+(\kappa-1) \cos \theta \sin \theta v_{z}\right]^{2}-\rho V_{s} v_{z}^{2} d \mathbf{x}
$$

which is negative. Thus, the energy is decreasing, which ensures the stability of the boundary value problem.

\section{Numerical illustration}

Let us consider an homogeneous $2 \mathrm{D}$ square domain and an elastic initial condition: $v(\mathbf{x}, 0)=v_{0}(\mathbf{x})$ with

$$
v_{0}(\mathbf{x})=\vec{\nabla} \times e^{-\pi^{2}\|\mathbf{x}\|^{2}}+\vec{\nabla} e^{-\pi^{2}\|\mathbf{x}\|^{2}}
$$

and $\underline{\underline{\sigma}}(\mathbf{x}, 0)=0$. Then, P-waves and a S-waves can be generated. In an isotropic medium (see Fig. 3), the magnitude of the velocity field depicts two circles, and it is observed at different times of simulation: $1 \mathrm{~s}$ at Fig. 3(a), $1.5 \mathrm{~s}$ at Fig. 3(b). After $6 \mathrm{~s}$ of simulation (see Fig. 3(c)), only spurious reflections which are caused 
by the ABCs, are noticeable. In a TTI medium (see Fig. 4), the magnitude of the velocity field describes a quasi-ellipsoid for the $\mathrm{P}$-wave and a quasi-square for the S-wave, see Fig 4. This is due to the (non-elliptic) TTI parameter choice. After $6 \mathrm{~s}$ of simulation, Fig. 4(c), only the spurious reflections caused by the ABCs are noticeable. The comparison with the isotropic case depicts the same kind of reflections, with the same intensity.

Let us underline that in this TTI case, the use of PMLs leads to an exponential growing of the numerical solution, until the simulation stops (within one second). A rigorous analysis of this phenomena can be found in [3].

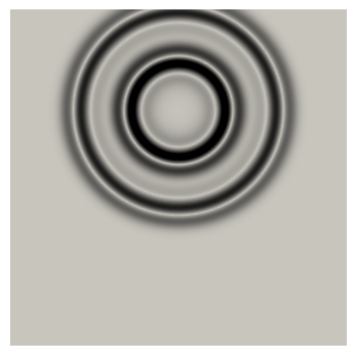

(a) Iso $1 \mathrm{~s}$.

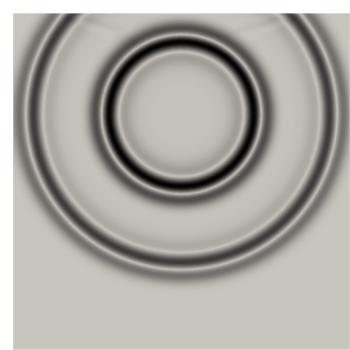

(b) Iso $1.5 \mathrm{~s}$.

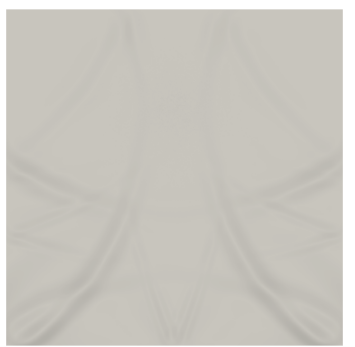

(c) Iso $6 \mathrm{~s}$.

FiguRE 3. Velocity magnitude at different time of the simulation

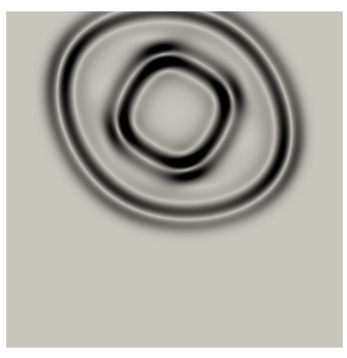

(a) TTI $1 \mathrm{~s}$.

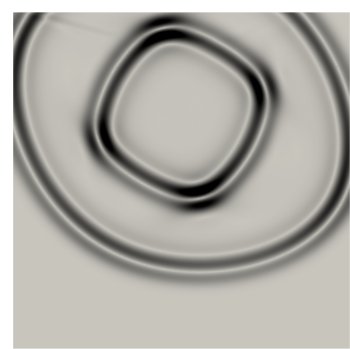

(b) TTI $1.5 \mathrm{~s}$.

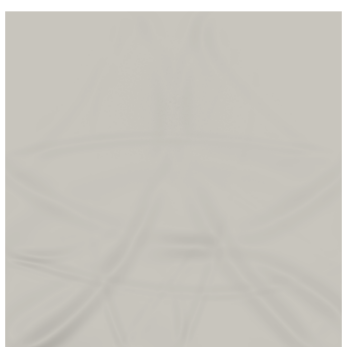

(c) TTI $6 \mathrm{~s}$.

FiguRE 4. Velocity magnitude at different time of the simulation

Now, let us compare the elliptic TTI ABC and the isotropic ABC efficiencies in the same general TTI elastic media (i.e. non-elliptic), see Fig. 5. The L2-energy is measured at each time step. The initial condition is a P-wave in Fig. 5(a), a S-wave in Fig. 5(b) and an elastic source in Fig. 5(c), so as to focus on the P-wave and $\mathrm{S}$-wave $\mathrm{ABC}$ reflections. In the three cases, the use of the anisotropic $\mathrm{ABC}$ clearly shows better results, in the sense that the pollution involved by the reflections is lower (about twice lower).

\section{CONCLUSION}

We have proposed a new $\mathrm{ABC}$ for 2D elliptic TTI elastic waves which outperforms the standard isotropic $\mathrm{ABC}$ in such media. Moreover, results show that the ABC also performs well in general TTI elastic media (i.e. non-elliptic). Besides, it generates some spurious reflections which can be considered as noise in a RTM/seismicimaging context. It is worth noting that these ABCs stay stable in anisotropic media, offering an alternative to PMLs. In the following, we work on an extension of our construction process to general TTI media. 


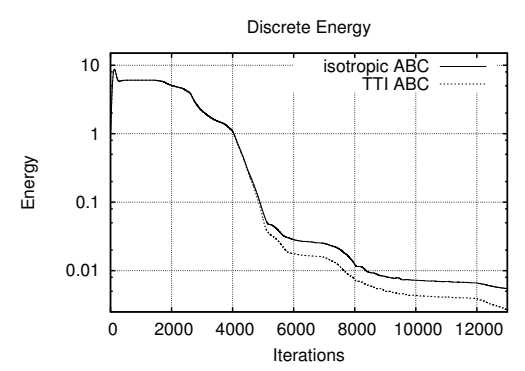

(a) P-wave

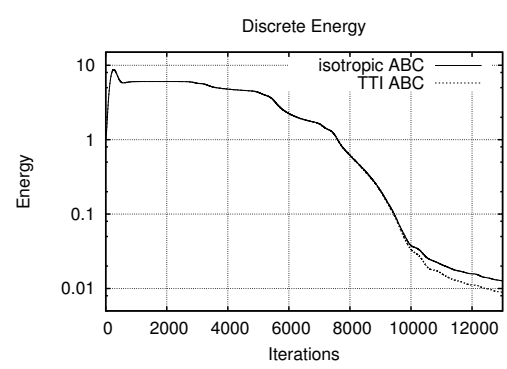

(b) S-wave

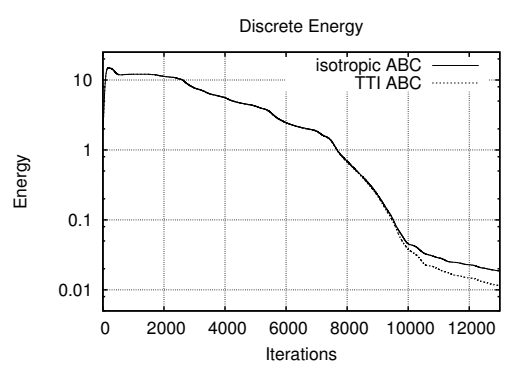

(c) PS-wave

FigURE 5. L2-energy with different initial condition

\section{REFERENCES}

[1] Antoine X. And Barucq H., Microlocal Diagonalization of Strictly Hyperbolic Pseudodifferential Systems and Application to the Design of Radiation Conditions in Electromagnetism, SIAM Journal on Applied Mathematics, 61:1877-1905, 2001.

[2] BÉcAche, E., Résolution par une méthode d'équations intégrales d'un problème de diffraction d'ondes élastiques transitoires par une fissure, PhD thesis, Paris VI, 1991.

[3] Bécache, E. and Fauqueux, S. and Joly, P., Stability of perfectly matched layers, group velocities and anisotropic waves, Journal of Computational Physics, 188(2):399-433, 2003.

[4] Bécache, E. and Givoli, D. And Hagstrom, T., High-order Absorbing Boundary Conditions for anisotropic and convective wave equations, Journal of Computational Physics, 229:1099-1129, 2010.

[5] Bérenger, J.-P., A perfectly matched layer for the absorption of electromagnetic waves, Journal of computational physics, 114:185-200, 1994.

[6] Bérenger, J.-P., Three-dimensional perfectly matched layer for the absorption of electromagnetic waves, J. of Comp. Phys. 127:363-379, 1996.

[7] Delcourte, S. and Fezoui, L. And Glinsky-Olivier, N., A high-order discontinuous Galerkin method for the seismic wave propagation, ESAIM: Proceedings, 27:70-89, 2009.

[8] Duveneck, E. And Bakker, M. P.,Stable P-wave modeling for reverse-time migration in tilted TI media, Geophysics, 76(2):65$75,2011$.

[9] Engquist, B. And Majda, A., Absorbing boundary conditions for the numerical simulation of waves, Mathematics of Computations, 31:629-651, 1977.

[10] Engquist, B. And Majda, A., Radiation boundary conditions for acoustic and elastic wave calculations, Comm. Pure Appl. Math., 32:314-358, 1979.

[11] Halpern, L., Étude de conditions aux limites absorbantes pour des schémas numériques relatifs a des équations hyperboliques linéaires, PhD thesis, Paris VI, 1980.

[12] Podgornova, O., Transparent Boundary Conditions for elastic anisotropic (VTI) media: axially symmetric case, Commun. Comput. Phys., 11(2):541-554, 2012.

[13] Reynolds, A. C., Boundary conditions for the numerical solution of wave equations problem, Geophy., 43(6):1099-1110, 1978.

[14] Savadatti, S. and Guddati, M.N., Absorbing boundary conditions for scalar waves in anisotropic media. Part 1: Time harmonic modeling, J. Comput. Phys., 229(19):6696-6714, 2010.

[15] Savadatti, S. And Guddati, M.N., Absorbing boundary conditions for scalar waves in anisotropic media. Part 2: Timedependent modeling, J. Comput. Phys., 229(18):6644-6662, 2010.

[16] Savadatti, S. and Guddati, M.N., Accurate absorbing boundary conditions for anisotropic elastic media. Part 1: Elliptic anisotropy, J. Comput. Phys., 231(2):7584-7607, 2012.

[17] Savadatti, S. And Guddati, M.N., Accurate absorbing boundary conditions for anisotropic elastic media. Part 2: Untitled non-elliptic anisotropy, J. Comput. Phys., 231(2):7608-7625, 2012.

[18] Thomsen, L., Weak elastic anisotropy, Geophysics, 51(10):1954-1966, 1986.

[19] Tsogka, C., Modélisation mathématique et numérique de la propagation des ondes élastiques tridimensionnelles dans des milieux fissurés, PhD thesis, Paris IX Dauphine, 1999.

[20] Tsvankin, I., Seismic signatures and analysis of reflection data in anisotropic media, Elsevier, Helbig K. and Tritel S., 2001. 